American Journal of Pharmaceutical Education 2016; 80 (8) Article 133.

\title{
RESEARCH
}

\section{Motivations and Predictors of Cheating in Pharmacy School}

\author{
Eric J. Ip, PharmD, Kathy Nguyen, PharmD, Bijal M. Shah, BPharm, PhD, \\ Shadi Doroudgar, PharmD, Monica K. Bidwal, PharmD \\ Touro University California College of Pharmacy, Vallejo, California \\ Submitted August 4, 2015; accepted October 7, 2015; published October 25, 2016.
}

Objective. To assess the prevalence, methods, and motivations for didactic cheating among pharmacy students and to determine predictive factors for cheating in pharmacy colleges and schools.

Methods. A 45-item cross-sectional survey was conducted at all four doctor of pharmacy programs in Northern California. For data analysis, $t$ test, Fisher exact test, and logistic regression were used.

Results. Overall, $11.8 \%$ of students admitted to cheating in pharmacy school. Primary motivations for cheating included fear of failure, procrastination, and stress. In multivariate analysis, the only predictor for cheating in pharmacy school was a history of cheating in undergraduate studies.

Conclusion. Cheating occurs in pharmacy schools and is motivated by fear of failure, procrastination, and stress. A history of past cheating predicts pharmacy school cheating. The information presented may help programs better understand their student population and lead to a reassessment of ethical culture, testing procedures, and prevention programs.

Keywords: pharmacy, medical, cheating, academic dishonesty, students

\section{INTRODUCTION}

Numerous studies have investigated academic dishonesty from various educational levels, ranging from elementary to graduate school. ${ }^{1-4}$ Academic dishonesty may begin as early as elementary school where children bend or break the rules to win a competition and increases in middle/junior high and high school..$^{5-7}$ Eighty-eight percent of undergraduate students also admit to some form of academic dishonesty. ${ }^{8}$ Reported motivations for academic dishonesty among high school and undergraduate students include competition, inadequate study time, difficult assignments, or lack of interest in course work. ${ }^{9}$ There is a continuum of academic dishonesty to professional health care schools.

The terms "academic dishonesty" and "cheating" will be used interchangeably in the current study depending on how the term is used in prior studies. Multiple studies describe cheating among medical students, with self-reported rates ranging from $0-58.2 \% .{ }^{10-12}$ Stimmel and Yens surveyed 114 medical school deans in the United States and Canada, and cheating was reported by $70 \%$ of US and $35 \%$ of Canadian medical schools. ${ }^{13}$ Examples of academic dishonesty in the didactic setting include but are not limited to copying another student's

Corresponding Author: Eric J. Ip, Touro University California College of Pharmacy, 1310 Club Drive, Vallejo, CA 94592. Tel: 707-638-5993. Fax: 707-638-5959. E-mail: eric.ip@tu.edu examination, bringing a cheat sheet to an examination, receiving assistance on a take home assignment/examination, stealing an examination, plagiarizing a paper, and falsifying results on a laboratory/research project. $^{10,12}$ Technological developments also contribute to academic misconduct and include outsourced/contract assignment writing, computerized watches (eg, smartwatches), programmable calculators, and online-based examinations (which may be difficult to invigilate remotely). Additionally, academic dishonesty is not limited to the didactic setting and may also occur during the clinical years (ie, reporting a laboratory test as pending when it has not been ordered or reporting a physical examination as normal when it was not performed). ${ }^{12}$ Academic dishonesty is particularly concerning among health care professions students as it may result in lower competence and continued unethical behavior beyond graduation. ${ }^{14}$

While academic dishonesty has been studied more extensively in medical school, much less is known about its occurrence in pharmacy school. Four studies have examined academic dishonesty in pharmacy programs. ${ }^{2,5,15,16}$ Rabi and colleagues surveyed pharmacy students from four universities located in the Midwest and Eastern United States and reported $16.3 \%$ of students admitted to "cheating" in pharmacy school. ${ }^{2}$ Additionally, more than half of the students believed that cheating was a part of life and that at least one student cheated on any given examination. ${ }^{2}$ Even greater numbers were seen in non-US pharmacy programs. Aggarwal and colleagues 


\section{American Journal of Pharmaceutical Education 2016; 80 (8) Article 133.}

surveyed students from two pharmacy schools in England and found $80 \%$ of the students admitted to at least one incident of academic dishonesty with a higher prevalence seen in males. ${ }^{15}$ Henning and colleagues surveyed pharmacy and medical students in New Zealand and found $91 \%$ engaged in copying, $34 \%$ in cheating, and $60 \%$ in collusion. Austin and colleagues surveyed Canadian pharmacy students and pharmacist educators and found that more than $90 \%$ of students admitted to academic dishonesty. ${ }^{16}$ While rates of academic dishonesty in pharmacy school have been reported in the literature, none of the studies delved into motivations or predictive factors.

The objectives of this study were to assess the prevalence, methods, and motivating factors for academic dishonesty among pharmacy students and to determine if certain predictive factors (eg, history of academic dishonesty, psychiatric conditions, or stress levels) may place a student at a higher risk. Information from this study may help pharmacy schools develop strategies and prevention programs to decrease academic dishonesty at their institution.

\section{METHODS}

This study was a 45-item cross-sectional survey conducted in person at all four doctor of pharmacy (PharmD) programs in Northern California (eg, Touro University California College of Pharmacy, University of the Pacific Thomas J. Long School of Pharmacy \& Health Sciences, California Northstate University College of Pharmacy, and University of California San Francisco School of Pharmacy). Three of the programs are private institutions and one is public. All four programs outline their academic integrity policies, typically in the form of a student handbook. Inclusion criteria consisted of pharmacy students in the second year of their didactic curriculum who were willing to take part in the survey. Second-year students were selected as they had completed at least one year of the didactic pharmacy program and were not yet on advanced pharmacy practice experiences (APPEs). Students who submitted incomplete surveys $(<80 \%$ of the survey complete) were excluded. Specific variables queried included: demographics (age, gender, ethnicity, marital status, and number of children), academic dishonesty (admitted cheating, methods, motivating factors, history), psychiatric diagnoses, and stress levels. ${ }^{2,5,15-17}$ Stress levels were measured by the Perceived Stress Scale (PSS), a validated instrument consisting of 10 multiplechoice questions scored on a 0 to 4 scale (with requisite reverse scoring for four items). ${ }^{18}$

A pharmacy student investigator recruited participants at each of the four Northern California pharmacy programs from November 4, 2014 to March 21, 2015.
Each pharmacy school was contacted to obtain consent to administer the survey. All four Northern California pharmacy schools agreed to allow student participation in the survey study, and their students were notified of the date, location, and purpose of the study via e-mail listserv. The pharmacy student investigator (rather than the pharmacy faculty co-investigators) administered the survey to enhance student participation and truthfulness. The survey was anonymous and administered using paper and pen; no identifying information was collected. To enhance participation, the survey was conducted in a regularly scheduled class period. Students deposited their completed survey inside a slot at the top of a sealed cardboard box. Upon depositing the completed survey in the box, students received a piece of candy as an incentive for taking the survey. The study received institutional review board approval from Touro University California.

Data analyses were conducted using STATA v12 (StataCorp LP, College Station, TX). As the survey was primarily descriptive in nature, means and standard deviations were reported for continuous data as a measure of central tendency while frequencies were reported for categorical data. Logistic regression analysis was used to identify risk factors for cheating in pharmacy school. A $p$ value of less than 0.05 was considered significant.

\section{RESULTS}

Out of a possible 525 students among the four schools, 452 students were in attendance and 360 responded to the survey. Twenty-nine surveys were excluded because they were incomplete $(n=15)$ or were left completely blank $(n=14)$. The exclusion of these subjects did not alter the significance of any of the survey questions. These exclusions resulted in a final analytic cohort of 331 students who completed the survey, resulting in a response rate of $63.0 \%$.

The demographics of the second-year pharmacy students from the schools are summarized in Table 1. The majority of the pharmacy students surveyed were 21-25 years old (59.7\%), female (65.1\%), Asian or Pacific Islander $(74.3 \%)$, single $(86.3 \%)$, and did not have any children/dependents (94.6\%). A 3.0-3.49 pharmacy school grade point average (GPA on a 4.0 scale) was the most common, and a majority of students $(63.6 \%)$ planned to apply to a postgraduate program. The mean Perceived Stress Score (PSS) for the students was 18.2 (maximum score of 40), which closely mirrored a nationally represented sample of pharmacy students (18.5). ${ }^{19}$

Table 2 describes the students' cheating history and knowledge of cheating incidents at their pharmacy school. When asked if they had ever cheated in pharmacy school, 11.8\% responded "Yes." The highest percentage 


\section{American Journal of Pharmaceutical Education 2016; 80 (8) Article 133.}

Table 1. Demographics of Pharmacy Students

\begin{tabular}{|c|c|}
\hline Variables & No. $(\%)$ \\
\hline \multicolumn{2}{|l|}{ Age } \\
\hline$\leq 20$ years old & $2(0.6)$ \\
\hline $21-25$ & $197(59.7)$ \\
\hline $26-29$ & $94(28.5)$ \\
\hline$\geq 30$ & $37(11.2)$ \\
\hline \multicolumn{2}{|l|}{ Gender } \\
\hline Female & $215(65.1)$ \\
\hline Male & $115(34.9)$ \\
\hline \multicolumn{2}{|l|}{ Ethnicity } \\
\hline Caucasian & $51(16.0)$ \\
\hline African American/Black & $5(1.6)$ \\
\hline Hispanic/Latino & $10(3.1)$ \\
\hline Asian/Pacific Islander & $237(74.3)$ \\
\hline Middle Eastern & $11(3.5)$ \\
\hline American Indian/Alaskan Native & $1(0.3)$ \\
\hline Other & $4(1.2)$ \\
\hline \multicolumn{2}{|l|}{ Marital status } \\
\hline Single & $284(86.3)$ \\
\hline Married & $31(9.4)$ \\
\hline Not married but living with a partner & $13(4.0)$ \\
\hline Divorced & $1(0.3)$ \\
\hline Widowed & $0(0)$ \\
\hline \multicolumn{2}{|l|}{ Children/dependents } \\
\hline Yes & $18(5.4)$ \\
\hline \multicolumn{2}{|l|}{ Pharmacy school GPA (4.0 scale) } \\
\hline$<2.5$ & $15(4.6)$ \\
\hline $2.5-2.99$ & $63(19.4)$ \\
\hline $3.0-3.49$ & $149(45.9)$ \\
\hline $3.5-4.0$ & $98(30.1)$ \\
\hline \multicolumn{2}{|l|}{$\begin{array}{l}\text { Planning to apply for postgraduate } \\
\text { program }\end{array}$} \\
\hline Yes & $210(63.6)$ \\
\hline $\begin{array}{l}\text { Perceived Stress Score (PSS) mean } \\
\quad(\mathrm{SD}), \text { range }^{\mathrm{a}}\end{array}$ & $18.2(6.3), 2-39$ \\
\hline
\end{tabular}

${ }^{a} A$ range of 0-40 is possible upon completing the Perceived Stress Scale (PSS) questionnaire

of admitted cheating occurred in middle school/junior high school (36.2\%), followed by high school (33.3\%), and then during undergraduate studies (18.1\%). More than half of the pharmacy students $(56.1 \%)$ were aware of student cheating incidents at their institution. Table 3 provides a breakdown of admitted pharmacy school cheating by each program. No significant differences were noted in admitted cheating between private and public pharmacy schools.

Table 4 describes specific examples of academic dishonesty. The most common forms of academic dishonesty pharmacy students admitted to included: handing down work to students in a lower year $(47.7 \%$ of students), copying another student's coursework with the student's permission ( $36.5 \%$ of students), offering details
Table 2. Cheating History and Awareness

\begin{tabular}{lr}
\hline Scenarios & No. (\%) \\
\hline Have you ever cheated in pharmacy school? & \\
$\quad$ Yes & $292(11.8)$ \\
$\quad$ No & \\
Have you ever cheated in undergraduate? & $60(18.1)$ \\
$\quad$ Yes & $271(81.9)$ \\
$\quad$ No & \\
Have you ever cheated in high school? & $110(33.3)$ \\
$\quad$ Yes & $220(66.7)$ \\
$\quad$ No & \\
Have you ever cheated in middle school/ & $120(36.2)$ \\
junior high? & $221(63.8)$ \\
Yes & \\
No & \\
Are you aware of any incident(s) of cheating & \\
$\quad$ involving pharmacy students at your & $185(56.1)$ \\
institution? & $145(43.9)$ \\
Yes & \\
No &
\end{tabular}

to a peer regarding content of an oral exam or objective structured clinical examination (OSCE) $(29.1 \%$ of students), asking a peer for details regarding content of an oral/practical examination or OSCE (26.6\% of students), and copying directly from a reference source without acknowledging the source or citing appropriately (16.5\% of students). A much smaller percentage of students reported cheating during an examination: $7.3 \%$ admitted copying from a neighbor, $3.7 \%$ asked someone for the answer, $0.9 \%$ used hidden notes, and $0.6 \%$ used an unauthorized electronic device during a written or electronic examination.

Students who admitted to cheating during pharmacy school $(n=39)$ were asked to rate various potential motivations for cheating using a 5-point Likert scale as described in Table 5. Highly rated motivations for cheating included fear of failure [score of 4.0 (1.1)], procrastination of study [score of 4.0 (1.0)], and stress [score of 3.5 (1.4)]. Financial pressure, peer pressure, parental/family pressure, illness, perception of unfair grading, and attaining entry to a postgraduate program were not highly rated motivations for cheating.

Students who admitted to cheating in pharmacy school were more than three times as likely to use prescription stimulants without a prescription than students who did not admit to cheating ( $12.8 \%$ vs $3.8 \%, p=0.014)$. A higher percentage of younger students (21-25 years old) admitted to cheating $(66.7 \%)$ compared to other age groups, however the result was not significant $(p=0.55)$. No significant differences were seen with gender, ethnicity, marital status, having children/dependents, pharmacy school GPA, plans to apply for a postgraduate program, 


\section{American Journal of Pharmaceutical Education 2016; 80 (8) Article 133.}

Table 3. Admitted Cheating by Each Pharmacy School

\begin{tabular}{lc}
\hline Pharmacy School & No. $(\%)$ \\
\hline 1 & $4 / 88(4.5)$ \\
2 & $18 / 102(17.6)$ \\
3 & $4 / 49(8.2)$ \\
4 & $13 / 92(14.1)$ \\
\hline
\end{tabular}

PSS scores, or psychiatric diagnoses between the two groups.

Potential risk factors for cheating in pharmacy school are summarized in Table 6. Cheating in undergraduate studies (OR 11.7, $p<0.001$ ) was the sole predictor of cheating in pharmacy school. Similarly, cheating in high school (OR 25.1, $p<0.001$ ) was the only predictor of cheating in undergraduate studies, and cheating in middle school (OR 29.4, $p<0.001$ ) was the only predictor of cheating in high school.

\section{DISCUSSION}

The current study is the second to demonstrate that academic dishonesty occurs among PharmD students in the United States. ${ }^{2}$ Just more than one in 10 students admitted to "cheating in pharmacy school," and even larger percentages were seen when specific examples of academic dishonesty were queried. The most common motivations for cheating in pharmacy school included fear of failure, studying procrastination, and stress. A history of cheating in undergraduate studies was the only predictor for cheating in pharmacy school.

Rates of admitted cheating in pharmacy school in the current study were similar to rates described by Rabi and colleagues of four Midwest or Eastern US pharmacy schools $(16.3 \%){ }^{2}$ Rates of admitted cheating in these two US studies were much lower than those found in England, New Zealand, and Canada. ${ }^{5,15,16}$ A possible reason for this is that all US pharmacy schools are currently doctoral programs while the three non-US studies mentioned above included baccalaureate or master's degree programs. The study by Baldwin and colleagues as well as the current study confirmed this notion as higher rates of cheating occurred in educational schooling preceding the doctoral degree (eg, middle school/junior high, high school, and undergraduate studies). ${ }^{10}$ Despite the lower incidence of admitted cheating in US pharmacy schools, the true prevalence is likely higher because of the sensitive nature of the topic, and academic dishonesty likely remains a concern for many institutions.

Pharmacy students more frequently admitted to performing specific forms of academic dishonesty than admitting to "cheating" in pharmacy school. These results were also reported by two other studies and suggest that students may not view certain academically dishonest behaviors as cheating and may even consider them the "norm.", 2,5 The most common forms of academic dishonesty in the current study were peer-based (ie, handing down work to students in lower years, copying another student's coursework with the student's permission, asking or offering details to a peer regarding an oral/practical examination or OSCE). Peer pressure or feeling loyalty to peers may be reasons for such peer-based academic dishonesty. ${ }^{10}$ Additionally, Emmerton and colleagues reported an ambiguity regarding students' interpretations of academic integrity, especially with regard to collusion. ${ }^{14}$ The $11.8 \%$ of reported cheating by the pharmacy students was likely interpreted as examination cheating based on the percentages of the following data: copied from a neighbor $(7.3 \%)$, asked someone for the answer $(3.7 \%)$, hidden notes $(0.9 \%)$, and unauthorized electronic device $(0.6 \%)$. Similar to the study by Rabi and colleagues, cheating on didactic examinations was less commonly reported and may be a result of the belief that students would eventually need to know the material (ie, when they take licensure examinations) and that cheating may only hurt that individual in the longrun. ${ }^{2,10}$ Other possible reasons may also include stringent invigilation or possible public humiliation if a student were to be caught cheating during an examination. Health care professions students who cheat in the classroom are more likely to cheat in actual patient care activities (ie, falsify patient information or report findings as normal despite not performing the procedure), which may carry on throughout the professional career. ${ }^{12}$

The primary motivating factors for cheating were fear of failure, studying procrastination, and higher stress levels. Fear of failure was also the most common motivating factor for cheating among medical students. ${ }^{20}$ To address fear of failure, studying habits, and stress management, pharmacy programs may benefit from investing more resources such as counselors, support groups, or peer teaching. ${ }^{17}$ Support groups and stress coping strategies have positive effects among undergraduate, medical, and nursing students and would likely be beneficial among pharmacy students as well. ${ }^{21,22}$

Similar to Rabi and colleagues, ${ }^{2}$ the current study found the only predictor of cheating in pharmacy school was cheating in undergraduate studies. Unlike studies by Aggarwal and colleagues and Henning and colleagues, the male gender was not a predictor of reported cheating in the current study. ${ }^{5,15}$ Similar to pharmacy school cheating, the only predictor of cheating in undergraduate studies was cheating in high school, and the only predictor of cheating in high school was cheating in middle school. Cheating behaviors that occur earlier in life seem to carry 


\section{American Journal of Pharmaceutical Education 2016; 80 (8) Article 133.}

Table 4. Specific Examples of Academic Dishonesty

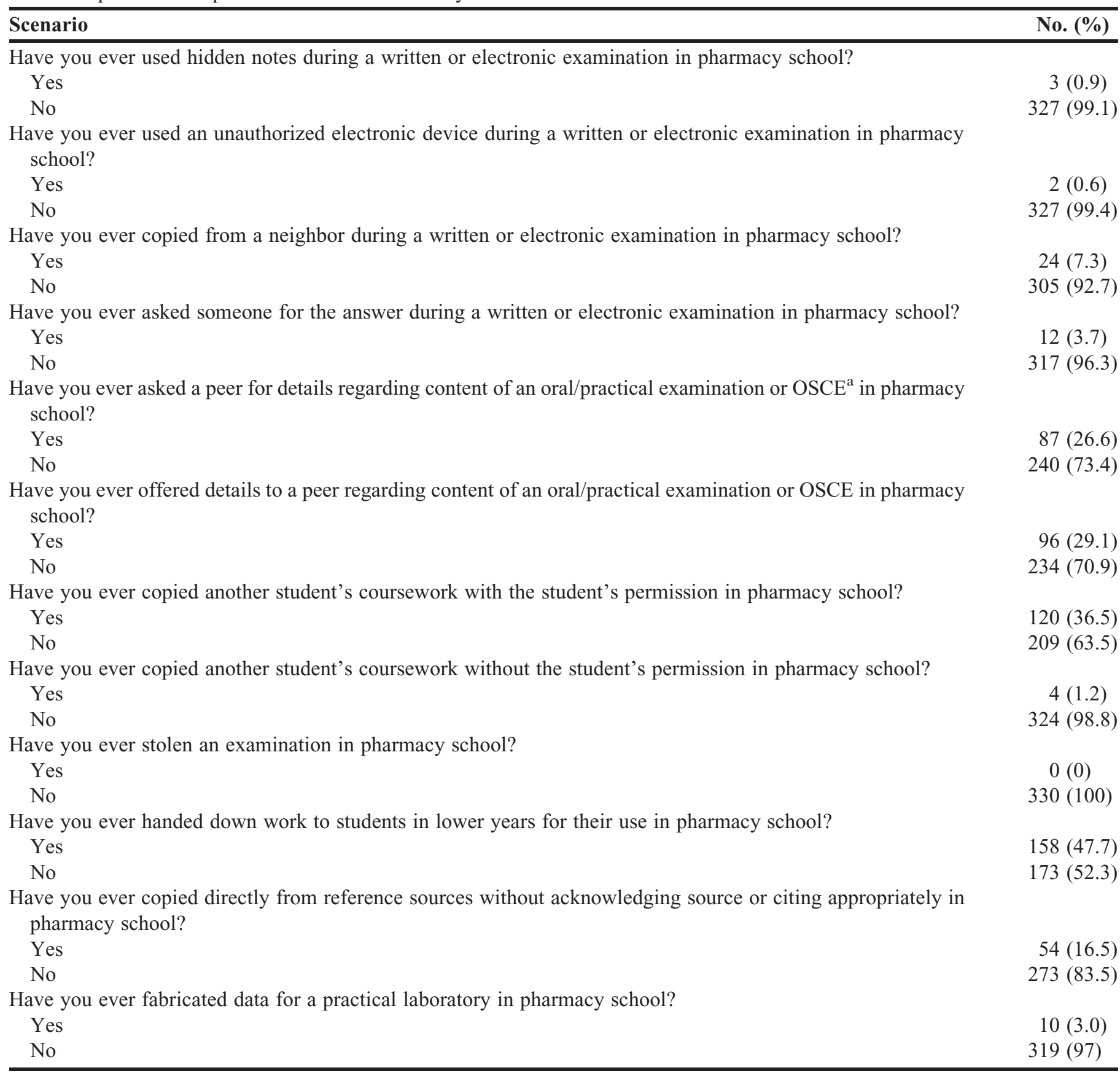

${ }^{\mathrm{a}}$ Objective Structured Clinical Examination

on for many individuals through subsequent phases of education. Callender and colleagues showed that kindergarteners who took part in severe cheating or covert rule violations were more likely to have behavioral problems during later childhood. ${ }^{23}$ Similarly, noncognitive traits and early social-emotional functioning displayed in childhood can predict personal and public health outcomes in adulthood (eg, education, employment, criminal activity, substance use, and mental health). ${ }^{24,25}$ Thus, parents and childhood teachers are key players in preventing cheating behavior later in life. ${ }^{23}$ Furthermore, cheating in the didactic setting increases unethical behavior in the clinical setting. ${ }^{12}$ Curbing academic dishonesty and promoting a culture of integrity should be a priority for pharmacy schools early on and reinforced as students progress throughout their education and upon entering professional practice.

Academic institutions have implemented various methods to decrease academic dishonesty and promote ethical behavior. ${ }^{2}$ Some health care professions schools 


\section{American Journal of Pharmaceutical Education 2016; 80 (8) Article 133.}

Table 5. Motivations for Cheating Among Pharmacy Students

\begin{tabular}{lc}
\hline Motivation & Score $^{\mathbf{a}}$ \\
\hline Fear of failure & $4.0(1.1)$ \\
Procrastination of study & $4.0(1.0)$ \\
Stress & $3.5(1.4)$ \\
Lack of confidence & $3.0(1.3)$ \\
Desire to maintain a high class standing & $3.0(1.4)$ \\
Lack of interest in course & $2.9(1.4)$ \\
Emotional problems & $2.7(1.6)$ \\
To get in to postgraduate residency/ fellowship/ & $2.5(1.4)$ \\
$\quad$ graduate program & \\
Unfair grading & $2.5(1.2)$ \\
Illness & $2.5(1.5)$ \\
Parental/family pressure & $2.2(1.3)$ \\
Peer pressure & $2.2(1.1)$ \\
Financial pressure & $2.1(1.2)$ \\
\hline Data are expressed as mean (SD) & \\
a Scores range from $1-5: 1=$ strongly disagree, $2=$ disagree, & \\
3=neutral, 4=agree, $5=$ strongly agree &
\end{tabular}

have moved to a pass/fail grading system to alleviate academic stress. ${ }^{20,26}$ Others have implemented an academic honor code, but data on its effectiveness in reducing cheating are mixed. ${ }^{10,13}$ Suggestions to reduce examination cheating include having sufficient proctors, paying attention to student gestures during a live examination, avoiding using the same examination (ie, creating authentic assessments), not leaving examinations unattended in an office, and not returning graded examinations to students. ${ }^{27,28}$ Additional methods include having inclass instead of at-home assessments, utilizing computerized examination testing which randomizes questions and answers (eg, ExamSoft), incorporating lower stakes assessments, limiting restroom breaks, and scheduling sufficiently spaced/timed assessments to reduce examination stress. To deter plagiarism, some institutions mandate completion of academic integrity training and utilize Internet-based plagiarism-prevention services (eg, www. turnitin.com). A culture of integrity and high ethical expectations led by administration, faculty, staff, and students should be implemented early upon matriculation and continued throughout the student's pharmacy career.
There is a large, and growing, body of literature regarding best assessment practices and their success requires a multifaceted approach. ${ }^{29}$ Whether this literature has been optimally applied to pharmacy assessments is unclear and would require further investigation.

There are several limitations to this study. First, all four colleges of pharmacy were located in Northern California and no other regions in the United States were included in the analysis. However, rates of cheating and types of academic dishonesty in this study were similar to the Midwest/Eastern United States study and limited data exists on this topic. Second, only second-year pharmacy students were surveyed, and patterns of academic dishonesty could only be detailed in the didactic setting, not a clinical setting. Third, surveys by nature result in a recall bias as subjects are required to recall past experiences. Fourth, because of the sensitive nature of academic dishonesty and potential fear of repercussions, the percentage of students admitting to cheating may be lower than the actual number, and cheating students may have omitted answering particular questions. Next, the demographics portion of the survey did not ask about prior enrollment (ie, experience as a university student could expose these participants to more methods of cheating) or about the culture of the university (ie, if "passing down" or "sharing" assignments/old examinations is commonplace). Finally, students may have different perceptions of what constitutes "cheating" in a self-report study, resulting in less accurate cheating rates. ${ }^{14}$ However, the current study queried a variety of academically dishonest behavior (Table 3 ) to provide a more detailed portrayal of actual behavior.

\section{CONCLUSION}

One in 10 students in the current sample of pharmacy schools admitted to cheating in pharmacy school. Peerbased forms of academic dishonesty were more common than examination cheating. Primary motivations included fear of failure, procrastination, and stress. Of the included variables, the sole predictor of cheating in pharmacy school was a history of cheating in undergraduate studies.

Table 6. Risk Factors for Admitted Cheating in Pharmacy School

\begin{tabular}{lcr}
\hline & Odds Ratio 95\% Confidence Interval & $p$ value \\
\hline Gender & $1.2,0.5-2.8$ & 0.66 \\
Cheating in middle school & $0.91,0.30-2.79$ & 0.88 \\
Cheating in high school & $2.29,0.63-8.27$ & 0.21 \\
Cheating in undergraduate & $11.69,4.41-31.03$ & $<0.001$ \\
Any psychiatric diagnosis & $0.53,0.11-2.50$ & 0.42 \\
Perceived Stress Score (PSS) & $1.06,0.99-1.13$ & 0.08 \\
\hline
\end{tabular}

${ }^{a}$ Includes major depressive disorder, bipolar disorder, an anxiety disorder, schizophrenia, and attention deficit-hyperactivity disorder 


\section{American Journal of Pharmaceutical Education 2016; 80 (8) Article 133.}

The information presented in this study may help pharmacy programs better understand their student population, which may lead to a reassessment of ethical culture, testing procedures, and prevention programs.

\section{REFERENCES}

1. Anderman EM, Midgley C. Changes in self-reported academic cheating across the transition from middle school to high school. Contemp Educ Psychol. 2004;29(4):499-517.

2. Rabi SM, Patton LR, Fjortoft N, Zgarrick DP. Characteristics, prevalence, attitudes, and perceptions of academic dishonesty among pharmacy students. Am J Pharm Educ. 2006;70(4):Article 73.

3. Murdock TB, Hale NM, Weber MJ. Predictors of cheating among early adolescents: academic and social motivations. Contemp Educ Psychol. 2001;26(1):96-115.

4. Whitley BE. Factors associated with cheating among college students: a review. Res High Educ. 1998;39(3):235-274.

5. Henning MA, Ram S, Malpas P, Shulruf B, Kelly F, Hawken SJ. Academic dishonesty and ethical reasoning: pharmacy and medical school students in New Zealand. Med Teach. 2013;35(6):e1211-e1217. 6. Kleiner C, Lord M. The cheating game. US News \& World Report. 1999;127(20):54-63.

7. James H. Honesty as a character trait among young people. J Educ Res. 1933;26(8):572-578.

8. Stern EB, Havlicek L. Academic misconduct: results of faculty and undergraduate student surveys. J Allied Health. 1986;15(2):129-142.

9. Cizek GJ. Cheating on Tests: How to Do It, Detect It, and Prevent It. London, UK: Routledge; 1999.

10. Baldwin Jr DC, Daugherty SR, Rowley BD, Schwarz MD.

Cheating in medical school: a survey of second-year students at 31 schools. Acad Med. 1996;71(3):267-273.

11. Dans PE. Self-reported cheating by students at one medical school. Acad Med. 1996;71(1):S70-72.

12. Sierles F, Hendrickx I. Cheating in medical school. Acad Med. 1980;55(2):124-125.

13. Stimmel B, Yens D. Cheating by medical students on examinations. Am J Med. 1982;73(2):160-164.

14. Emmerton L, Jiang H, McKauge L. Pharmacy students' interpretation of academic integrity. Am J Pharm Educ. 2014;78(6): Article 119.

15. Aggarwal R, Bates I, Davies JG, Khan I. A study of academic dishonesty among students at two pharmacy schools. Pharm J. 2002;269(7219):529-533.
16. Austin Z, Simpson S, Reynen E. "The fault lies not in our students, but in ourselves": academic honesty and moral development in health professions education - results of a pilot study in Canadian pharmacy. Teach High Educ. 2005;10(2):143-156.

17. Bidwal MK, Ip EJ, Shah BM, Serino MJ. Stress, drugs, and alcohol use among health care professional students: a focus on prescription stimulants. J Pharm Pract. 2015;28(6):535-542.

18. Cohen S, Kamarck T, Mermelstein R. A global measure of perceived stress. J Health Soc Behav. 1983;24(4):385-396.

19. Votta RJ, Benau EM. Predictors of stress in doctor of pharmacy students: results from a nationwide survey. Curr Pharm Teach Learn. 2013;5(5):365-372.

20. Cockayne TW, Samuelson Jr C. The crime and punishment of cheating in medical school. Proc Annu Conf Res Med Educ. 1983;22:166-171.

21. Jain S, Shapiro SL, Swanick S, et al. A randomized controlled trial of mindfulness meditation versus relaxation training: effects on distress, positive states of mind, rumination, and distraction. Ann Behav Med. 2007;33(1):11-21.

22. Luo Y, Wang H. Correlation research on psychological health impact on nursing students against stress, coping way and social support. Nurse Educ Today. 2009;29(1):5-8.

23. Callender KA, Olson SL, Kerr DC, Sameroff AJ. Assessment of cheating behavior in young school-age children: distinguishing normative behaviors from risk markers of externalizing psychopathology. J Clin Child Adolesc Psychol. 2010;39(6):776-788. 24. Jones DE, Greenberg M, Crowley M. Early social-emotional functioning and public health: the relationship between kindergarten social competence and future wellness. Am J Public Health. 2015;105 (11):2283-2290.

25. Moffitt TE, Arseneault L, Belsky D, et al. A gradient of childhood self-control predicts health, wealth, and public safety. Proc Natl Acad Sci USA. 2011;108(7):2693-2698.

26. Reed DA, Shanafelt TD, Satele DW, et al. Relationship of pass/ fail grading and curriculum structure with well-being among preclinical medical students: a multi-institutional study. Acad Med. 2011;86(11):1367-1373.

27. Brown DL. Cheating must be okay - everybody does it! Nurse Educ. 2002;27(1):6-8.

28. Moore MG, Savrock JT. Distance education in the health sciences. readings in distance education, number 8. ERIC; 2001. 29. Boyd P, Bloxham S. Developing Effective Assessment In Higher Education: A Practical Guide. New York, NY: McGraw-Hill Educationd; 2007. 